\title{
Surgical strategies for older patients with glioblastoma
}

\author{
Tanyeri Barak ${ }^{1,2,8} \cdot$ Shaurey Vetsa ${ }^{1,2} \cdot$ Arushii Nadar ${ }^{1,2} \cdot$ Lan Jin $^{1,2,3,4} \cdot$ Trisha P. Gupte $^{1,2} \cdot$ Elena I. Fomchenko ${ }^{1,2}$. \\ Danielle F. Miyagishima ${ }^{1,2}$. Kanat Yalcin ${ }^{1}$ - Sagar Vasandani ${ }^{1,2}$. Evan Gorelick ${ }^{1,2}$. Amy Y. Zhao ${ }^{1,2}$. \\ Joseph Antonios $^{1,2} \cdot$ Brianna Carusillo Theriault $^{1,2} \cdot$ Nathan Lifton $^{1,2}$. Neelan Marianayagam ${ }^{1,2} \cdot$ Bulent Omay $^{1,2}$. \\ Zeynep Erson Omay ${ }^{1,2} \cdot$ Anita Huttner ${ }^{2,5} \cdot$ Declan McGuone $^{2,5} \cdot$ Nicholas A. Blondin $^{2,6} \cdot$ Zachary Corbin $^{2,6}$. \\ Robert K. Fulbright ${ }^{2,7} \cdot$ Jennifer Moliterno ${ }^{1,2}$
}

Received: 17 July 2021 / Accepted: 29 September 2021 / Published online: 9 October 2021

(c) The Author(s), corrected publication 2021

\begin{abstract}
Objective While adjuvant treatment regimens have been modified for older patients with glioblastoma (GBM), surgical strategies have not been tailored.

Methods Clinical data of 48 consecutive patients aged 70 years or older, who underwent surgical resection for GBM with intraoperative ultrasonography (IoUS) alone or combination with intraoperative MRI (IoMRI) at Yale New Haven Hospital were retrospectively reviewed. Variables were analyzed, and comparative analyses were performed.

Results The addition of IoMRI was not superior to IoUS alone in terms of overall survival $(\mathrm{OS})(\mathrm{P}=0.306)$, Karnofsky Performance Score (KPS) at postoperative 6 weeks $(\mathrm{P}=0.704)$ or extent of resection $(\mathrm{P}=0.263)$. Length of surgery $($ LOSx $)$, however, was significantly longer $(\mathrm{P}=0.0002)$ in the IoMRI group. LOSx $(\mathrm{P}=0.015)$ and hospital stay $(\mathrm{P}=0.025)$ were predictors of postoperative complications. Increased EOR (GTR or NTR) $(\mathrm{P}=0.030)$, postoperative adjuvant treatment ( $\mathrm{P}$ $<0.0001)$ and postoperative complications $(\mathrm{P}=0.006)$ were predictive for OS. Patients with relatively lower preoperative KPS scores $(<70)$ showed significant improvement at postoperative 6 weeks $(\mathrm{P}<0.0001)$. Patients with complications $(\mathrm{P}=$ 0.038) were more likely to have lower KPS at postoperative 6 weeks.

Conclusions Aggressive management with surgical resection should be considered in older patients with GBM, even those with relatively poor KPS. The use of ioMRI in this population does not appear to confer any measurable benefit over ioUS in experienced hands, but prolongs the length of surgery significantly, which is a preventable prognostic factor for impeding care.
\end{abstract}

Keywords Glioblastoma $\cdot$ Surgical strategies $\cdot$ Neuronavigation $\cdot$ Intraoperative imaging

\section{Introduction}

Glioblastoma (GBM) is the most common primary malignant brain tumor in adults. The incidence of GBM increases with advanced age, with a peak in the 75-84 age group [1].

Jennifer Moliterno

jennifer.moliternogunel@yale.edu

1 Department of Neurosurgery, Yale School of Medicine, 15 York St., LLCI 810, CT 06520-8082 New Haven, USA

2 Yale Brain Tumor Center, Smilow Cancer Hospital, CT, New Haven, USA

3 Department of Surgery, Yale School of Medicine, New Haven, USA

4 Department of Environmental Health Sciences, Yale School of Public Health, New Haven, USA
Studies show that more than one fourth of GBM patients are older than 70 years of age at the time of diagnosis [2]. Recent studies centered on the use of radiation and chemotherapy in this older population have garnered results and recommendations to help specifically guide treatment of

5 Department of Pathology, Yale School of Medicine, New Haven, USA

6 Department of Neurology, Yale School of Medicine, New Haven, USA

7 Department of Radiology and Biomedical Imaging, Yale School of Medicine, New Haven, USA

8 Department of Genetics, Yale School of Medicine, New Haven, Connecticut, USA 
GBM [3-8] in this population, however strategies for surgical management have yet to be optimized.

Though there is compelling evidence that maximal safe resection and chemoradiation are associated with improved survival in younger GBM patients, many physicians have traditionally followed more conservative approaches when treating septuagenarians and octogenarians [9-11]. This paradigm, however, is recently challenged by a growing body of evidence demonstrating that elderly patients benefit from maximal safe resection and chemoradiation $[3,4,5,6,7,8$, $12,13,14,15,16,17,18,19,20]$. Indeed, improved survival with increased EOR in older GBM patients occurs in patients without postoperative complications [21] and prolonged surgery is an independent predictor of complications in elderly patients undergoing craniotomies [22-29].

More sophisticated intraoperative image guidance techniques have been used more routinely in GBM surgery to achieve maximal EOR. Though the use of intraoperative MRI (IoMRI) may now be considered the "gold standard", it prolongs surgical time [30]. On the other hand, IoUS remains a reliable, real-time imaging tool, which improves surgical resection [31-35], while reducing the length of surgery (LOSx) compared with IoMRI. Surgeon expertise with its interpretation, however, is important for its success. Given the notion that increased surgical time could potentially be associated with postoperative complications, we sought to determine whether there were indeed any differences in outcome with these intraoperative image guidance techniques in older patients with GBM.

\section{Methods}

\section{Clinical data acquisition}

This study was approved by the Yale School of Medicine Institutional Review Board. Forty-eight consecutive patients, age 70 years and older, who underwent surgical resection by the senior author for histologically confirmed GBM between November 2015 and January 2021 were included. Demographic and clinical variables were retrospectively collected. All patients were followed until death or through submission of this manuscript with none lost during follow-up. Patients not meeting the aforementioned criteria and/or those who underwent biopsy were excluded.

\section{Functional assessment}

Functional assessment was done using the Karnofsky Performance Scale (KPS) score, as determined by retrospective review of detailed medical records. Preoperative KPS was determined at the time of admission, while postoperative KPS was assessed at approximately 6 weeks follow-up.
Functional improvement was determined based on the difference. To further evaluate the clinical improvement of patients, we also used The Neurologic Assessment in NeuroOncology (NANO) scale [36, 37].

\section{Tumor location, volume and extent of resection}

Tumor volume was calculated using the modified ellipsoidal formula, $V=(4 / 3) \times \pi \times\left(D_{1} / 2\right) \times\left(D_{2} / 2\right) \times\left(D_{3} / 2\right)$ based on the maximum tumor diameters in perpendicular dimensions. All patients underwent contrast enhanced T1-weighted MRI before surgery and within $48 \mathrm{~h}$ after surgery when possible and were used to determine the amount of tumor removed. All surgeries were performed by the senior author (JM). The extent of resection (EOR) was calculated using the formula 1 - [((Pre-Op Tm Volume - Post-Op Tm Volume) / PreOp Tm Volume) $* 100]$. In accordance with prior literature, EOR was independently determined by a board certified neuroradiologist (RKF) as: (1) gross total resection (i.e. GTR: $\geq 98 \%$ tumor removal), (2) near total resection (i.e. NTR: $90-98 \%$ tumor removal) or (3) subtotal resection (i.e. STR: $<90 \%$ of tumor removal) [38]. For patients who were unable to undergo postoperative MRI, EOR was determined based on intraoperative imaging, post-operative $\mathrm{CT}$ and operative report.

\section{Intraoperative image guidance techniques}

The use of the Brainlab Neuronavigation system and an operating microscope was standard in all surgeries. The 3D intraoperative ultrasound (IoUS) system (BK Flex Focus 800 or bk5000 Neurosurgical System) and 3T intraoperative magnetic resonance imaging (IoMRI) (3T MRI Scanner, Siemens MAGNETOM) were used as intraoperative image guidance modalities.

\section{Tumor pathology}

Histological diagnosis of all tumors was determined by board-certified neuropathologists in accordance with WHO guidelines. MGMT status was determined in forty-seven patients (Table 1).

\section{Statistical analysis}

Statistical analyses were performed using SPSS (IBM SPSS Statistics, version 24) or Graphpad Prism version 8.3.0. Univariate analyses used either simple logistic regression model or one of the following: Fisher Exact test (categorical variables when two groups were compared); pairwise Fisher exact test (if more than two groups were compared); t-test with Welch correction (continuous variables with normal distribution); Mann Whitney-U test was conducted for any 
Table 1 General patient characteristics and comparative analysis between ioUS and combined ioMRI + ioUS groups

\begin{tabular}{|c|c|c|c|c|}
\hline Characteristics & Total & IoUS & ioUS+IoMRI & $P$ value \\
\hline Age in Years $($ Mean \pm SD) & $75.98 \pm 4.99$ & $80.16 \pm 5.99$ & $74.43 \pm 3.36$ & 0.0053 \\
\hline \multicolumn{5}{|l|}{ Gender } \\
\hline Female & 20 & $3(23.08 \%)$ & 17 & \multirow[t]{2}{*}{0.188} \\
\hline Male & 28 & $10(76.92 \%)$ & 18 & \\
\hline \multicolumn{5}{|l|}{ Presentation } \\
\hline Altered mental status & $17(35.42 \%)$ & $5(38.46 \%)$ & $12(34.29 \%)$ & 1 \\
\hline Facial strength & $9(18.75 \%)$ & $2(15.38 \%)$ & $7(20 \%)$ & 1 \\
\hline \multicolumn{5}{|l|}{ Language/Speech deficit } \\
\hline Seizures & $5(10.42 \%)$ & $2(15.38 \%)$ & $3(8.57 \%)$ & 0.602 \\
\hline Motor deficit and Ataxia & $16(33.33 \%)$ & $6(46.15 \%)$ & $10(28.57 \%)$ & 0.311 \\
\hline Visual field cut & $10(20.83 \%)$ & $1(7.69 \%)$ & $9(25.71 \%)$ & 0.248 \\
\hline Past medical history & $44(91.67 \%)$ & $12(92.31 \%)$ & $32(91.43 \%)$ & 1 \\
\hline Hypertension & $27(56.25 \%)$ & $7(53.85 \%)$ & $20(57.14 \%)$ & 1 \\
\hline Diabetes Mellitus & $9(18.75 \%)$ & $1(7.69 \%)$ & $8(22.86 \%)$ & 0.411 \\
\hline Hyperlipidemia & $23(47.92 \%)$ & $6(46.1 \%)$ & $17(48.57 \%)$ & 1 \\
\hline Thyroid disorder & $5(10.42 \%)$ & 0 & $5(14.29 \%)$ & 0.304 \\
\hline DVT/PE & $4(8.33 \%)$ & $1(7.69 \%)$ & $3(8.57 \%)$ & 1 \\
\hline Cardiovascular Disorders & $17(35.42 \%)$ & $8(61.54 \%)$ & $9(25.71 \%)$ & 0.039 \\
\hline Arrhythmia & $9(18.75 \%)$ & $4(30.77 \%)$ & $5(14.29 \%)$ & 0.228 \\
\hline Stroke & $4(8.33 \%)$ & $2(15.38 \%)$ & $2(5.71 \%)$ & 0.294 \\
\hline CAD & $4(8.33 \%)$ & $2(15.38 \%)$ & $2(5.71 \%)$ & 0.294 \\
\hline Aortic aneurysm & $3(6.25 \%)$ & $3(23.08 \%)$ & 0 & 0.017 \\
\hline Aortic stenosis & $2(4.16 \%)$ & $1(7.69 \%)$ & $1(2.86 \%)$ & 0.473 \\
\hline \multicolumn{5}{|l|}{ Localization } \\
\hline Right & $32(66.67 \%)$ & $7(53.85 \%)$ & $25(71.43 \%)$ & \multirow[t]{2}{*}{0.311} \\
\hline Left & $16(33.33 \%)$ & $6(46.15 \%)$ & $10(28.57 \%)$ & \\
\hline \multicolumn{5}{|l|}{ Anatomic classification } \\
\hline Supratentorial & $47(97.92 \%)$ & $13(100 \%)$ & $34(97.14 \%)$ & \multirow[t]{2}{*}{1} \\
\hline Infratentorial & $1(2.08 \%)$ & 0 & $1(2.86 \%)$ & \\
\hline \multicolumn{5}{|l|}{ Location } \\
\hline Frontal & $15(31.25 \%)$ & $4(30.77 \%)$ & $11(31.43 \%)$ & 1 \\
\hline Temporal & $22(45.83 \%)$ & $6(46.15 \%)$ & $16(45.71 \%)$ & 1 \\
\hline Parietal & $16(33.33 \%)$ & $5(38.46 \%)$ & $11(31.43 \%)$ & 0.735 \\
\hline Occipital & $3(6.25 \%)$ & $1(7.69 \%)$ & $2(5.71 \%)$ & 1 \\
\hline Multilobar & $8(16.67 \%)$ & $3(23.08 \%)$ & $5(14.29 \%)$ & 0.665 \\
\hline Multifocal & $1(2.08 \%)$ & $1(7.69 \%)$ & 0 & 0.271 \\
\hline Eloquent structure involvement & $16(33.33 \%)$ & $5(38.46 \%)$ & $11(31.43 \%)$ & 0.735 \\
\hline Basal Ganglia & $6(12.50 \%)$ & $3(23.08 \%)$ & $3(8.57 \%)$ & 0.323 \\
\hline Insula & $8(16.67 \%)$ & $1(7.69 \%)$ & $7(20 \%)$ & 0.418 \\
\hline Thalamus & $3(6.25 \%)$ & $2(15.38 \%)$ & $1(2.86 \%)$ & 0.265 \\
\hline Motor cortex & $1(2.08 \%)$ & $1(7.69 \%)$ & 0 & 0.271 \\
\hline Large arterial encasement by the tumor & $1(2.08 \%)$ & 0 & $1(2.86 \%)$ & 1 \\
\hline Brainstem & $1(2.08 \%)$ & 0 & $1(2.86 \%)$ & 1 \\
\hline Corpus callosum & 0 & 0 & 0 & NA \\
\hline Median tumor volume & 27.35 & 24.35 & 34.25 & 0.1454 \\
\hline \multicolumn{5}{|l|}{ MGMT status } \\
\hline Methylated & $17(36.17 \%)$ & $3(6.38 \%)$ & $14(29.79 \%)$ & \multirow[t]{3}{*}{$1^{*}$} \\
\hline Partially methylated & $6(12.77 \%)$ & $3(6.38 \%)$ & $3(6.38 \%)$ & \\
\hline Non-Methylated & $24(51.06 \%)$ & $6(12.77 \%)$ & $18(36.30 \%)$ & \\
\hline
\end{tabular}

Bold values indicate statistical significance at the $\mathrm{p}<0.05$ level

* Methylated and partially methylated MGMT have been grouped together for the comparative analysis

$D V T$ Deep venous thrombosis, $P E$ Pulmonary embolus 
variables, which did not pass a Kolmogorov-Smirnov normality test; Wilcoxon Matched-Pairs Signed Rank test was used for paired variables with normal distribution. When the dependent variable was continuous, univariate and multivariate analyses were performed using linear regression models. For multivariate analysis, a binary logistic regression with a forward stepwise selection model was conducted using all variables tested in univariate analysis with an entry $P$ value threshold 0.05 and removal threshold 0.1 . Cox proportional hazards model was used to examine the impact of the variables on overall survival (OS). Kaplan Meier survival curves were performed to visualize OS for significant variables. $\mathrm{P}$ values $<0.05$ were considered statistically significant.

\section{Results}

\section{Cohort characteristics}

Forty-seven patients had newly diagnosed GBM; one patient who presented with a "recurrent" GBM was operated on in another hospital 5 months prior to undergoing surgery with the senior author and had not received any adjuvant treatment. The average age was 75.98 years (range: 70-92). The median preoperative KPS score was 70 (range: 20-100), while the median KPS at 6-weeks follow-up was 90 (range: 20-100). The median tumor volume was $27.35 \mathrm{~cm} 3$. These results are summarized in Tables 1 and 2.

GTR or NTR were achieved in forty-three cases (89.58\%). Average length of hospital stay was 4.58 days, with $47.92 \%$ of patients being discharged home. Twenty-four patients (50\%) received adjuvant treatment according to Stupp Protocol [39], five patients (10.42\%) according to Perry Protocol [3] and eight patients (16.67\%) received hypo-fractionated radiotherapy alone. Eleven patients $(22.92 \%)$ did not receive any adjuvant treatment.

\section{Comparison of intraoperative imaging modalities}

A combination of IoMRI and IoUS was used in 35 cases, while IoUS solely was used in 13 cases. There were no statistically significant differences between the IoUS and IoUS+IoMRI groups in terms of gender (Fisher Exact test, $\mathrm{P}=0.188$ ), pre-operative tumor volume (Mann-Whitney test, $\mathrm{P}=0.15$ ), involvement of eloquent structures (Fisher Exact Test; $\mathrm{P}=0.74$ ), MGMT status (Fisher Exact test, $\mathrm{P}=1$ ) or preoperative KPS score (Mann-Whitney test, $P=0.96)$. Patients in the IoUS group, however, were significantly older ( $\mathrm{t}$-test with Welch correction, $\mathrm{P}=0.005$ ) (Table 1).

While there was no difference in EOR (Pairwise Fisher Exact test, $\mathrm{P}=0.263$ ) between the groups, the LOSx was significantly longer (t-test with Welch correction,
$\mathrm{P}=0.0002)$ in the IoMRI group. There were no significant differences however with regards to postoperative complications (Fisher Exact test, $\mathrm{P}=0.42$ ), length of hospital stay (Mann-Whitney test, $\mathrm{P}=0.80$ ) or place of disposition (Pairwise Fisher Exact Test, $\mathrm{P}=0.918$ ). There were no differences in terms of number of patients who received adjuvant treatment with Stupp Protocol [39] (Fisher Exact test, $\mathrm{P}=0.193$ ), Perry Protocol [3] (Fisher Exact test, $\mathrm{P}=0.304$ ), hypo-fractionated radiotherapy alone (Fisher Exact test, $\mathrm{P}=0.187$ ) or who did not receive any adjuvant treatment (Fisher Exact test, $\mathrm{P}=0.14$ ) (Table 2). Patients in both groups showed significant functional improvement at 6 weeks postoperative follow-up (Wilcoxon matchedpairs signed Rank Test, $\mathrm{P}=0.039$ and $\mathrm{P}=0.0003$ in ioUS and ioMRI+ioUS, respectively).

\section{Postoperative complications}

Postoperative complications were observed in 8 patients (16.67\%), some of whom experienced more than one issue. Several of these complications were related to medical care, including urinary tract infections and pulmonary embolus (Table 2). Prolonged surgery time $(\mathrm{OR}=1.023$, 95\%, CI 1.005-1.043; $\mathrm{P}=0.013)$, pre-operative KPS score less than $70(\mathrm{OR}=11.67,95 \%$ CI $1.305-104.337$; $\mathrm{P}=0.028)$ and prolonged hospital stay $(\mathrm{OR}=1.249,95 \%$ CI 1.025-1.522; $\mathrm{P}=0.028$ ) were among the significant predictors of complications.

The frequency of postoperative complications in the ioMRI+ioUS group was more than two-times as much as the ioUS-alone cases (20-7.69\%), though this difference was not statistically significant (Fisher Exact Test, $\mathrm{P}=0.42)$.

LOSx $(\mathrm{OR}=1.033,95 \% \mathrm{CI} 1.006-1.06 ; \mathrm{P}=0.015)$ and hospital stay $(\mathrm{OR}=1.422,95 \% \mathrm{CI} 1.046-1.932 ; \mathrm{P}=0.025)$ remained significant in multiple logistic regression analysis (Table 3), suggesting that longer time spent in surgery and the hospital increases the likelihood of postoperative complications.

\section{Discharge disposition}

We analyzed the place of disposition as a reflection of immediate postoperative functional outcome. 23 patients (47.92\%) were discharged home, while 16 patients (33.33\%) were discharged to short-term skilled nursing facilities (SNF) and $9(18.75 \%)$ to rehabilitation centers. The multiple logistic regression model demonstrated that patients with a preoperative KPS score less than $70(\mathrm{OR}=0.211,95 \% \mathrm{CI}$ $0.061-0.732 ; \mathrm{P}=0.014$ ) were less likely to be discharged home and more likely to SNF or rehabilitations centers. 
Table 2 Descriptive analysis of the surgical outcome and comparison between the ioUS and combined ioMRI + ioUS group

\begin{tabular}{|c|c|c|c|c|}
\hline Characteristics & Total & IoUS & ioUS+IoMRI & $\mathrm{P}$ value \\
\hline \multicolumn{5}{|l|}{ Preoperative Karnofski performance scale (KPS) Score } \\
\hline$\geq 70$ & $26(54.17 \%)$ & $8(61.54 \%)$ & $18(51.43 \%)$ & \multirow[t]{2}{*}{0.746} \\
\hline$<70$ & $22(45.83 \%)$ & $5(38.46 \%)$ & $17(48.57 \%)$ & \\
\hline Preoperative NANO scale (median) & 3 & 2.5 & 3 & 0.917 \\
\hline \multicolumn{5}{|l|}{ Extent of resection } \\
\hline Gross total resection (GTR) & $31(64.58 \%)$ & $7(53.84 \%)$ & $24(68.58 \%)$ & 0.498 \\
\hline Near total resection (NTR) & $12(25 \%)$ & $3(23.08 \%)$ & $9(25.71 \%)$ & 1 \\
\hline Subtotal resection (STR) & $5(10.42 \%)$ & $3(23.08 \%)$ & $2(5.71 \%)$ & 0.115 \\
\hline Average length of surgery (minutes) & $169.7 \pm 58.93$ & $111.1 \pm 51.32$ & $189.7 \pm 47.11$ & 0.0002 \\
\hline Postoperative complications * & $8(16.67 \%)$ & $1(7.69 \%)$ & $7(20 \%)$ & 0.418 \\
\hline Altered mental status & $4(8.33 \%)$ & 0 & $4(11.43 \%)$ & 0.563 \\
\hline Urinary tract infection & $3(6.25 \%)$ & 0 & $3(8.57 \%)$ & 0.553 \\
\hline Wound infection & $2(4.17 \%)$ & 0 & $2(5.71 \%)$ & 1 \\
\hline Ileus & $2(4.17 \%)$ & 0 & $2(5.71 \%)$ & 1 \\
\hline Postoperative hematoma (Managed without evacuation) & $1(2.08 \%)$ & $1(7.69 \%)$ & 0 & 0.271 \\
\hline Pulmonary embolus & $1(2.08 \%)$ & 0 & $1(2.86 \%)$ & 1 \\
\hline Perforator infarct & $1(2.08 \%)$ & 0 & $1(2.86 \%)$ & 1 \\
\hline Atrial fibrillation & $1(2.08 \%)$ & 0 & $1(2.86 \%)$ & 1 \\
\hline Average length of the hospital stay after the surgery (Days) & $4.583 \pm 3.451$ & $4.769 \pm 2.891$ & $4.514 \pm 3.673$ & 0.803 \\
\hline \multicolumn{5}{|l|}{ Disposition } \\
\hline Home & $23(47.92 \%)$ & $6(46.15 \%)$ & $17(48.57 \%)$ & 1 \\
\hline Skilled nursing facilities (Short-term) & $16(33.33 \%)$ & $5(38.46 \%)$ & $11(31.43 \%)$ & 0.735 \\
\hline Rehabilitation centers & $9(18.75 \%)$ & $2(15.38 \%)$ & $7(20 \%)$ & 1 \\
\hline \multicolumn{5}{|l|}{ Functional outcome (Median) } \\
\hline Postoperative KPS score (at discharge) & 70 & 70 & 70 & 0.964 \\
\hline Postoperative follow-up KPS score (at 6 weeks follow-up) & 90 & 80 & 90 & 0.382 \\
\hline Median Follow-up NANO scale & 1 & 1 & 1 & 0.722 \\
\hline \multicolumn{5}{|l|}{ Changes in functional outcome } \\
\hline Improvement & $28(58.33 \%)$ & $6(46.15 \%)$ & $22(62.86 \%)$ & 0.339 \\
\hline Unchanged & $12(25 \%)$ & $5(38.46 \%)$ & $7(20 \%)$ & 0.263 \\
\hline Worsening & $8(16.67 \%)$ & $2(15.38 \%)$ & $6(17.14 \%)$ & 1 \\
\hline \multicolumn{5}{|l|}{ Chemoradiation protocols } \\
\hline Stupp protocol & $24(50 \%)$ & $4(30.77 \%)$ & $20(57.14 \%)$ & 0.193 \\
\hline Perry protocol & $5(10.42 \%)$ & 0 & $5(14.29 \%)$ & 0.304 \\
\hline Hypofractionated radiation treatment alone & $8(16.67 \%)$ & $4(30.77 \%)$ & $4(11.43 \%)$ & 0.187 \\
\hline No therapy & $11(22.92 \%)$ & $5(38.46 \%)$ & $6(17.14 \%)$ & 0.14 \\
\hline
\end{tabular}

Bold values indicate statistical significance at the $p<0.05$ level

*Some patients developed more than one complication

\section{Follow-up KPS score at postoperative six week and functional improvement}

We next sought to elucidate the variables contributing to the follow-up KPS score at postoperative 6 weeks. Univariate analysis implicated postoperative complications $(\mathrm{OR}=0.15$, 95\% CI 0.029-0.764; $\mathrm{P}=0.022$ ), and preoperative language deficit $(\mathrm{OR}=0.194,95 \% \mathrm{CI} 0.046-0.824 ; \mathrm{P}=0.026)$ as significant predictors of follow-up KPS score. In multivariate logistic regression model, postoperative complications
$(\mathrm{OR}=0.161,95 \% \mathrm{CI} 0.03-0.904 ; \mathrm{P}=0.038)$ and preoperative language deficit $(\mathrm{OR}=0.208,95 \%$ CI $0.045-0.971$; $\mathrm{P}=0.046$ ) remained significant to predict a follow-up KPS score less than 70 at postoperative 6 weeks.

We further analyzed the factors associated with postoperative functional improvement, which we defined as an at least 10-point difference between the follow-up KPS and the preoperative KPS scores. We found that 28 patients $(58.33 \%)$ showed functional improvement, while 12 patients (25\%) remained the same and 8 patients $(16.67 \%)$ worsened. 
Table 3 Logistic regression analysis of postoperative complications

\begin{tabular}{lrc}
\hline Characteristics & Odds Ratio (95\% CI) & P value \\
\hline Univariate analysis simple logistic regression & & \\
Length of surgery & $1.023(1.005-1.043)$ & $\mathbf{0 . 0 1 3}$ \\
Pre-operative KPS (KPS score $\geq 70$ vs. KPS score <70) & $0.085(0.010-0.767)$ & $\mathbf{0 . 0 2 8}$ \\
Length of hospital stay & $1.249(1.025-1.522)$ & $\mathbf{0 . 0 2 8}$ \\
Tumor volume & $0.974(0.934-1.016)$ & 0.226 \\
Pre-operative language deficit & $2.4(0.471-12.22)$ & 0.292 \\
Gender & $0.36(0.075-1.727)$ & 0.202 \\
Image guidance technique (ioUS vs. ioMRI + ioUS) & $0.333(0.037-3.014)$ & 0.328 \\
Pre-operative motor deficit & $1.246(0.258-6.031)$ & 0.784 \\
Age & $0.961(0.810-1.139)$ & 0.644 \\
Pre-operative altered mental status & $0.556(0.099-3.113)$ & 0.504 \\
Involvement of eloquent structures & $1.246(0.257-6.031)$ & 0.784 \\
Stepwise-Forward Multiple Logistic Regression & & \\
Length of surgery & $1.033(1.006-1.06)$ & $\mathbf{0 . 0 1 5}$ \\
Length of hospital stay & $1.422(1.046-1.932)$ & $\mathbf{0 . 0 2 5}$ \\
\hline
\end{tabular}

Bold values indicate statistical significance at the $\mathrm{p}<0.05$ level

KPS Karnofski Performance Scale; ioUS, intraoperative ultrasonography, ioMRI intraoperative magnetic resonance imaging. NANO, The Neurologic Assessment in Neuro-Oncology
Interestingly, $59 \%$ of patients with a preoperative KPS score less than 70 showed a follow-up KPS score 70 or higher and showed significant functional improvement (Wilcoxon matched-pairs signed Rank Test, $\mathrm{P}<0.0001$ ), although patients with a preoperative KPS score 70 or higher did not show a significant change $(\mathrm{P}=0.196)$ (Fig. 1). This result remained the same $(\mathrm{P}=0.1578)$ when we excluded two patients with a preoperative KPS score of 100 from the analysis. Taken together, these results suggest that patients with relatively lower preoperative KPS scores can demonstrate significant functional improvement following surgery, while those with higher scores maintain them.

Forty-three patients, who had clear physical examination records necessary for all components of scoring were assessed using the NANO scale. Median preoperative and follow-up NANO scale scores were 3 and 1, respectively. A significant improvement was observed when the functional status of patients was evaluated using NANO scale ( $\mathrm{P}=0.0036$, Wilcoxon Matched-Pairs Signed Rank test) (Fig. 1). $28(65 \%)$ of the patients showed at least 1 level improvement in NANO scale, while 20 patients $(46.5 \%)$ showed at least 2 levels, and $10(23.3 \%)$ patients showed at least 3 levels improvement.

\section{Postoperative adjuvant treatment}

We next elucidated variables contributing to the likelihood patients went on to receive postoperative adjuvant treatment, including the Stupp protocol [39], Perry protocol [3] or hypofractionated RT only.
We found that a preoperative KPS score 70 or higher showed the most significant association in univariate analysis (OR $=8.307,95 \%$ CI 1.557-44.32; P = 0.013) with more likelihood to proceed with adjuvant therapy, which was followed by the KPS score at postoperative 6 weeks $(\mathrm{OR}=5.143,95 \%$ CI 1.214-21.795; $\mathrm{P}=0.026)$.

Preoperative KPS score was significantly associated with postoperative adjuvant treatment in multivariate analysis $(\mathrm{OR}=16.251,95 \%$ CI 2.048-128.944; $\mathrm{P}=0.008)$. However, when we adjusted the analysis for the postoperative KPS score, we found that the preoperative KPS score is not a significant predictor of postoperative adjuvant treatment for the patients who had a follow-up KPS score less than 70 $(\mathrm{P}=0.99)$, underscoring the importance of postoperative KPS score in decision-making for postoperative adjuvant treatment.

These findings suggest that the functional status of older GBM patients after surgery is an important determinant in whether patients are able to continue with postoperative adjuvant treatment, and not necessarily predictive pre-operatively.

\section{Survival analyses}

Two patients were excluded: One who was operated in another institution for GBM five months prior to the second surgery in our institution was excluded, and another with COVID19 during the radiation treatment, which resulted in interruption of the treatment and death soon thereafter.

Univariate analysis showed the presence of postoperative complications $(\mathrm{HR}=4.119,95 \%$ CI 1.753-9.679; 
Fig. 1 Patients with pre-operative KPS score $<70$ demonstrated significant improvement 6-weeks after surgical resection. Paired parallel axis dot plot showing significant functional improvement for patients

with pre-op KPS score $<70$ following the surgical resection $(\mathrm{p}<0.0001)(\mathbf{A})$, while no significant changes were observed in pre-op KPS score $\geq 70$ group (B). Preoperative NANO scale improved significantly at postoperative follow-up assessment $(\mathrm{P}=0.0036)$ from a preoperative median score of 3 to 1 at follow-up (C). Abbreviations: Pre-op, preoperative; post-op, postoperative; KPS, Karnofski Performance Scale; NANO,

The Neurologic Assessment in Neuro-Oncology
A

\section{Pre-OP KPS score $<70$ \\ $P<0.0001$}

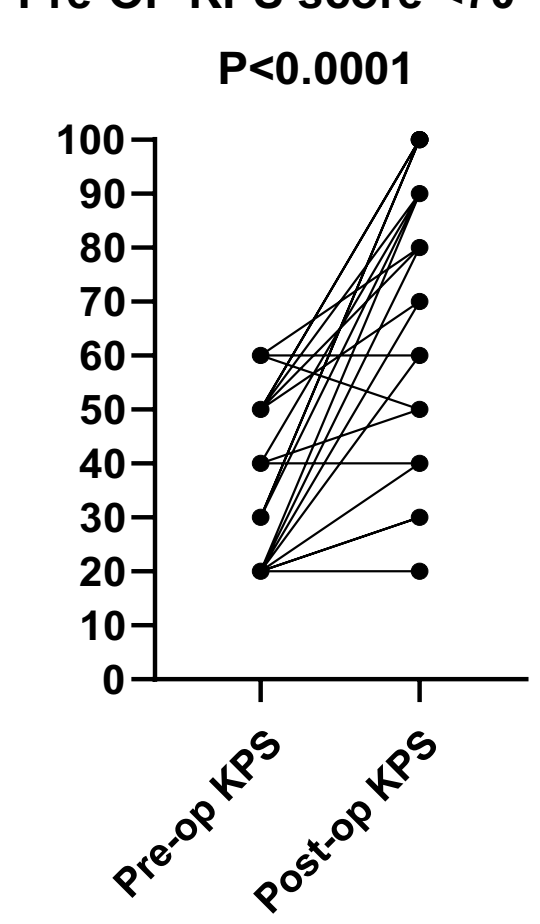

B

Pre-OP KPS score $\geq 70$

$P=0.196$
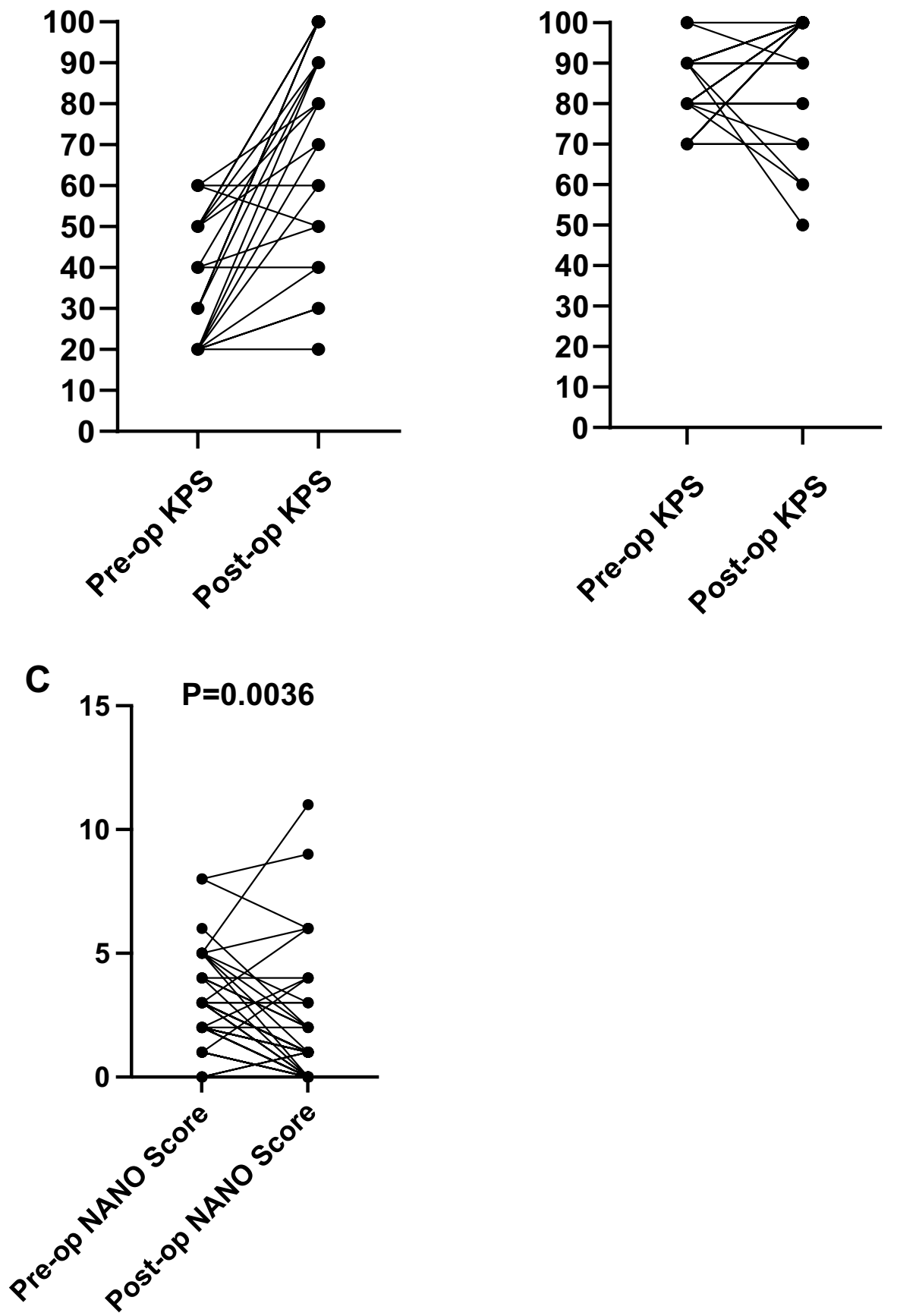

$\mathrm{P}=0.001)$ was predictive for overall survival (OS). In this cohort, we found that postoperative adjuvant therapy (any aforementioned type) is associated with improved OS compared to none (HR $=0.075,95 \%$ CI $0.028-0.204$, $\mathrm{P}<0.0001)$.
In multivariate analysis, we found that greater EOR (GTR or NTR vs. STR) (Median OS $=281$ vs. 163 days; $\mathrm{HR}=0.329,95 \%$ CI $0.121-0.896 ; \mathrm{P}=0.030)$ and use of postoperative adjuvant treatment (Median OS $=335$ vs. $110 ; \mathrm{HR}=0.144,95 \%$ CI $0.057-0.362 ; \mathrm{P}<0.0001)$, are associated with improved OS in this population of patients. 
Table 4 Univariate and Multivariate Analysis of overall survival with Cox proportional hazards model

\begin{tabular}{lll}
\hline Characteristics & Hazard ratio (95\% CI) & P value \\
\hline Univariate analysis & & \\
Adjuvant treatment & $0.075(0.028-0.204)$ & $\mathbf{3 . 4 7 e - 7}$ \\
Post-operative complications & $4.119(1.753-9.679)$ & $\mathbf{0 . 0 0 1}$ \\
Pre-operative KPS (KPS score $\geq 70$ vs. KPS score <70) & $0.559(0.301-1.040)$ & 0.066 \\
EOR (GTR+NTR vs. STR) & $0.540(0.209-1.400)$ & 0.205 \\
MGMT * (methylated and partially methylated vs. unmethylated) & $0.694(0.366-1.317)$ & 0.264 \\
Gender & $1.431(0.762-2.690)$ & 0.265 \\
Image guidance technique (ioUS vs. ioMRI + ioUS) & $1.450(0.712-2.954)$ & 0.306 \\
Pre-operative altered mental status & $1.290(0.677-2.460)$ & 0.439 \\
Involvement of eloquent structures & $1.192(0.621-2.289)$ & 0.598 \\
Pre-operative motor deficit & $1.175(0.606-2.281)$ & 0.633 \\
Tumor volume & $0.998(0.990-1.008)$ & 0.802 \\
Follow-Up KPS (KPS score $\geq 70$ vs. KPS score <70) & $1.044(0.495-2.203)$ & 0.910 \\
Multivariate analysis & & \\
EOR (GTR+NTR vs. STR) & & $\mathbf{0 . 0 3 0}$ \\
Post-operative complications & $0.329(0.121-0.896)$ & $\mathbf{0 . 0 0 6}$ \\
Adjuvant treatment & $3.620(1.433-9.144)$ & $\mathbf{3 . 8 0 e - 5}$ \\
\hline Bold values
\end{tabular}

Bold values indicate statistical significance at the $\mathrm{p}<0.05$ level

*An independent univariate analysis was further performed to assess the impact of MGMT status on overall survival in patients who had MGMT status determined as "methylated" or "unmethylated" and received temozolomide treatment $(\mathrm{n}=29),(\mathbf{P}=\mathbf{0 . 1 7 2}, \mathrm{HR}: 0.550,95 \%$ CI $0.233-1.30)$

Abbreviations: KPS, Karnofski Performance Scale; ioUS, intraoperative ultrasonography; ioMRI, intraoperative magnetic resonance imaging; EOR, Extent of resection; GTR, gross total resection; NTR, near total resection; STR, subtotal resection
Overall survival, however, was significantly shorter with the presence of postoperative complications (Median OS = 120 vs. 305 days; $\mathrm{HR}=3.620,95 \%$ CI $1.433-9.144$, $\mathrm{P}=0.006)($ Table 4$)$.

\section{Discussion}

GBM remains a challenging disease with an overall poor prognosis despite the most aggressive, multimodal treatment. Surgery, followed by chemoradiation, can be quite demanding, particularly for older individuals, therefore raising the question of how aggressive to be. Paradigms have been modified with regards to other aspects of GBM treatment in this population $[3,39]$, and thus we sought to determine whether surgical strategies can be similarly optimized.

In support of previous reports [4-6, 12-19], we demonstrated that older patients with GBM who undergo maximal EOR and adjuvant chemoradiation have an improved OS, suggesting that surgical resection and postoperative adjuvant treatment should not be withheld from this patient population. Patients with relatively lower pre-op KPS scores $(<70)$ were more likely to be discharged to short-term facilities but showed significant improvement at postoperative 6-weeks. In fact, 59\% of these patients received a follow-up KPS score of 70 or more at that time, which was a prognostic threshold important for predicting whether patients received postoperative adjuvant treatment. Indeed, patients who received postoperative chemoradiation, tolerated the treatment well and demonstrated improved OS compared to patients who did not receive adjuvant treatment. Thus, older patients, even those with relatively low preoperative KPS, benefit from surgery and can improve from their baseline preoperative functional state to tolerate adjuvant treatment and reap the OS benefits. Survival and quality of life outcomes, however, were significantly impacted by the presence of preoperative language deficit and postoperative complications, the latter of which was predictive for poor OS and follow-up KPS score at postoperative 6-weeks.

Given the prognostic implications of postoperative complications, and the trend that they increase with longer surgical time, surgical techniques and intraoperative adjuncts should be carefully considered to minimize complication risk, while aiming for maximal EOR. Similar to others, when investigating the usefulness of the addition of ioMRI, we found it does not offer any advantage for OS or functional improvement in this population. Indeed, a recent meta-analysis compared IoUS, IoMRI and other image guidance techniques in high grade glioma surgery and similarly found no significant difference between these modalities in terms of EOR and survival, while ioMRI use was significantly more expensive [31]. However, these studies did not compare 
clinical outcome in older patients. Indeed, ioMRI use in older patients in our cohort, significantly prolonged surgery, which served as a risk factor for postoperative complications in these patients, which were predictive of follow-up KPS score at postoperative 6-weeks and poor overall survival. There was a trend toward an increased frequency of postoperative complications in combined ioMRI+ioUS group compared to ioUS-alone cases (20-7.69\%) despite the latter group being older on average. Though this difference did not reach statistical significance, possibly due to sample size, this finding suggests that surgery in older patients with GBM should be aimed at minimizing the operative time and avoiding the use of unnecessary adjuncts. In experienced hands, ioUS can garner similar benefits as IoMRI, while potentially avoiding deleterious ones. While this is the strategy now employed by the senior author, it should be noted that older patients should be assessed on a case-by-case basis such that the addition of ioMRI could be justified in certain patients and circumstances.

Our study has limitations. First, it is retrospective and of a single center designed with a homogenous, but relatively small patient cohort. This could explain why no survival benefit with regards to MGMT status or pre-op KPS was observed, as well as the small number of those patients undergoing STR. Second, as expected, a significant association was found between EOR and improved OS in multivariate analysis, but oddly not observed in univariate analysis. While this may seem counterintuitive, other variables that impact OS (e.g., complications) may act as negative confounders for the association between GTR and OS in multivariate analysis, rendering the significance of EOR in multivariate, but not univariate analysis. As described before, the inclusion of negative confounders in the model would push the adjusted association away from the null, leading to the significance of EOR more significant in multivariate analysis [40]. Additionally, it is important to note that the average age of the patients in ioUS group was significantly older than the combined group. This difference was due to the senior's author intention to reduce the duration of surgeries in this relatively more senior age group, based on our hypothesis. However, the relatively older age in the ioUS group, would render that group at a disadvantage from a prognostic standpoint, which further underscores the observation. This study can serve as a pilot for data building the reasoning for further prospective studies with larger study cohorts.

\section{Conclusions}

Aggressive surgical resection improves OS and functional outcomes in older patients with GBM, and therefore maximal interventions should be considered in this patient population. In experienced hands, ioMRI use does not add any significant benefit over ioUS in terms of OS and functional outcomes in older GBM patients, but does increase the length of surgery, a preventable prognostic factor for postoperative complications.

Supplementary Information The online version contains supplementary material available at https://doi.org/10.1007/s11060-021-03862-z.

Acknowledgements We are grateful to the patients who contributed to this study. This study was supported by the Gregory M. Kiez and Mehmet Kutman Foundation. This study was also supported by the Connecticut Brain Tumor Alliance.

Open Access This article is licensed under a Creative Commons Attribution 4.0 International License, which permits use, sharing, adaptation, distribution and reproduction in any medium or format, as long as you give appropriate credit to the original author(s) and the source, provide a link to the Creative Commons licence, and indicate if changes were made. The images or other third party material in this article are included in the article's Creative Commons licence, unless indicated otherwise in a credit line to the material. If material is not included in the article's Creative Commons licence and your intended use is not permitted by statutory regulation or exceeds the permitted use, you will need to obtain permission directly from the copyright holder. To view a copy of this licence, visit http://creativecommons.org/licenses/by/4.0/.

\section{References}

1. Ostrom QT, Cioffi G, Gittleman H et al (2019) CBTRUS statistical report: primary brain and other central nervous system tumors diagnosed in the United States in 2012-2016. Neuro-oncology 21(Supplement_5):v1-v100

2. Fabbro-Peray P, Zouaoui S, Darlix A et al (2019) Association of patterns of care, prognostic factors, and use of radiotherapy-temozolomide therapy with survival in patients with newly diagnosed glioblastoma: a French national population-based study. J NeuroOncol 142(1):91-101

3. Perry JR, Laperriere N, O'Callaghan CJ et al (2017) Short-course radiation plus temozolomide in elderly patients with glioblastoma. New England J Med 376(11):1027-1037

4. Keime-Guibert F, Chinot O, Taillandier L et al (2007) Radiotherapy for glioblastoma in the elderly. New England J Med 356(15):1527-1535

5. Roa W, Brasher P, Bauman G et al (2004) Abbreviated course of radiation therapy in older patients with glioblastoma multiforme: a prospective randomized clinical trial. J Clin Oncol 22(9): $1583-1588$

6. Roa W, Kepka L, Kumar N et al (2015) International Atomic Energy Agency randomized phase III study of radiation therapy in elderly and/or frail patients with newly diagnosed glioblastoma multiforme. J Clin Oncol 33(35):4145-4150

7. Curran WJ Jr, Scott CB, Horton J et al (1993) Recursive partitioning analysis of prognostic factors in three radiation therapy oncology group malignant glioma trials. JNCI: J Nat Cancer Institute 85(9):704-710

8. Malmström A, Grønberg BH, Marosi C et al (2012) Temozolomide versus standard 6-week radiotherapy versus hypofractionated radiotherapy in patients older than 60 years with glioblastoma: the Nordic randomised, phase 3 trial. The Lancet Oncology 13(9):916-926 
9. Iwamoto FM, Reiner AS, Nayak L, Panageas KS, Elkin EB, Abrey LE (2009) Prognosis and patterns of care in elderly patients with glioma. Cancer 115(23):5534-5540

10. Barnholtz-Sloan JS, Williams VL, Maldonado JL et al (2008) Patterns of care and outcomes among elderly individuals with primary malignant astrocytoma. J Neurosurg 108(4):642-648

11. Lutterbach J, Bartelt S, Momm F, Becker G, Frommhold H, Ostertag C (2005) Is older age associated with a worse prognosis due to different patterns of care? A long-term study of 1346 patients with glioblastomas or brain metastases. Cancer: Interdisciplinary International Journal of the American Cancer Society 103(6):1234-1244

12. Almenawer SA, Badhiwala JH, Alhazzani W et al (2015) Biopsy versus partial versus gross total resection in older patients with high-grade glioma: a systematic review and meta-analysis. Neurooncology 17(6):868-881

13. Babu R, Komisarow JM, Agarwal VJ et al (2016) Glioblastoma in the elderly: the effect of aggressive and modern therapies on survival. Journal of neurosurgery 124(4):998-1007

14. Chaichana KL, Chaichana KK, Olivi A et al (2011) Surgical outcomes for older patients with glioblastoma multiforme: preoperative factors associated with decreased survival. Journal of neurosurgery 114(3):587-594

15. Noorbakhsh A, Tang JA, Marcus LP et al (2014) Gross-total resection outcomes in an elderly population with glioblastoma: a SEER-based analysis. Journal of neurosurgery 120(1):31-39

16. Vuorinen V, Hinkka S, Färkkilä M, Jääskeläinen J (2003) Debulking or biopsy of malignant glioma in elderly people-a randomised study. Acta neurochirurgica 145(1):5-10

17. Chaichana KL, Garzon-Muvdi T, Parker S et al (2011) Supratentorial glioblastoma multiforme: the role of surgical resection versus biopsy among older patients. Annals of surgical oncology 18(1):239-245

18. Iwamoto FM, Cooper AR, Reiner AS, Nayak L, Abrey LE (2009) Glioblastoma in the elderly: the Memorial Sloan-Kettering Cancer Center Experience (1997-2007). Cancer: Interdisciplinary International Journal of the American Cancer Society 115(16):3758-3766

19. Oszvald Á, Güresir E, Setzer M et al (2012) Glioblastoma therapy in the elderly and the importance of the extent of resection regardless of age. Journal of neurosurgery 116(2):357-364

20. Yin A-a, Zhang L-h, Cheng J-x et al (2013) Radiotherapy plus concurrent or sequential temozolomide for glioblastoma in the elderly: a meta-analysis. PLoS One 8(9):e74242

21. Karsy M, Yoon N, Boettcher L et al (2018) Surgical treatment of glioblastoma in the elderly: the impact of complications. Journal of neuro-oncology 138(1):123-132

22. Karhade AV, Fandino L, Gupta S et al (2017) Impact of operative length on post-operative complications in meningioma surgery: a NSQIP analysis. Journal of neuro-oncology 131(1):59-67

23. Golebiowski A, Drewes C, Gulati S, Jakola AS, Solheim O (2015) Is duration of surgery a risk factor for extracranial complications and surgical site infections after intracranial tumor operations? Acta neurochirurgica 157(2):235-240

24. Valentini LG, Casali C, Chatenoud L, Chiaffarino F, Uberti-Foppa C, Broggi G (2008) Surgical site infections after elective neurosurgery: a survey of 1747 patients. Neurosurgery 62(1):88-96

25. Oh T, Safaee M, Sun MZ et al (2014) Surgical risk factors for post-operative pneumonia following meningioma resection. Clinical neurology neurosurgery 118:76-79
26. Chibbaro S, Di Rocco F, Makiese O et al (2011) Neurosurgery and elderly: analysis through the years. Neurosurgical Review 34(2):229-234

27. Khaldi A, Helo N, Schneck MJ, Origitano TC (2011) Venous thromboembolism: deep venous thrombosis and pulmonary embolism in a neurosurgical population. J Neurosurgery 114(1):40-46

28. Browd SR, Ragel BT, Davis GE, Scott AM, Skalabrin EJ, Couldwell WT (2004) Prophylaxis for deep venous thrombosis in neurosurgery: a review of the literature. Neurosurgical Focus 17(4):1-6

29. Procter LD, Davenport DL, Bernard AC, Zwischenberger JB (2010) General surgical operative duration is associated with increased risk-adjusted infectious complication rates and length of hospital stay. J Am College Surg 210(1):60-65

30. Schroeck H, Welch TL, Rovner MS, Johnson HA, Schroeck FR (2019) Anesthetic challenges and outcomes for procedures in the intraoperative magnetic resonance imaging suite: a systematic review. J Clini Anesthesia 54:89-101

31. Eljamel MS, Mahboob SO (2016) The effectiveness and costeffectiveness of intraoperative imaging in high-grade glioma resection; a comparative review of intraoperative ALA, fluorescein, ultrasound and MRI. Photodiagnosis Photodynamic Therapy 16:35-43

32. Coburger J, Scheuerle A, Kapapa T et al (2015) Sensitivity and specificity of linear array intraoperative ultrasound in glioblastoma surgery: a comparative study with high field intraoperative MRI and conventional sector array ultrasound. Neurosurg Rev 38(3):499-509

33. Mahboob S, McPhillips R, Qiu Z et al (2016) Intraoperative ultrasound-guided resection of gliomas: a meta-analysis and review of the literature. World Neurosurg 92:255-263

34. Saether C, Torsteinsen M, Torp S, Sundstrøm S, Unsgård G, Solheim O (2012) Did survival improve after the implementation of intraoperative neuronavigation and 3D ultrasound in glioblastoma surgery? A retrospective analysis of 192 primary operations. J Neurol Surg Part A: Central European Neurosurg 73(02):073-078

35. Bander ED, Magge R, Ramakrishna R (2018) Advances in glioblastoma operative techniques. World Neurosurg 116:529-538

36. Nayak L, DeAngelis LM, Brandes AA et al (2017) The neurologic assessment in neuro-oncology (NANO) scale: a tool to assess neurologic function for integration into the response assessment in neuro-oncology (RANO) criteria. Neuro-oncology 19(5):625-635

37. Ung TH, Ney DE, Damek D et al (2019) The Neurologic Assessment in Neuro-Oncology (NANO) scale as an assessment tool for survival in patients with primary glioblastoma. Neurosurgery 84(3):687-695

38. Abhinav K, Aquilina K, Gbejuade H, La M, Hopkins K, Iyer V (2013) A pilot study of glioblastoma multiforme in elderly patients: treatments, O-6-methylguanine-DNA methyltransferase (MGMT) methylation status and survival. Clinical Neurol Neurosurg 115(8):1375-1378

39. Stupp R, Mason WP, Van Den Bent MJ et al (2005) Radiotherapy plus concomitant and adjuvant temozolomide for glioblastoma. New England J med 352(10):987-996

40. Mehio-Sibai A, Feinleib M, Sibai TA, Armenian HK (2005) A positive or a negative confounding variable? A simple teaching aid for clinicians and students. Annals of Epidemiol 15(6):421-423

Publisher's Note Springer Nature remains neutral with regard to jurisdictional claims in published maps and institutional affiliations. 